\title{
Mineração de dados aplicada à classificação automática de gêneros musicais
}

\section{Data mining applied to automatic music genres classification}

\author{
Paulo Sergio da Conceição Moreira ${ }^{\circledR}, 1$ and Denise Fukumi Tsunoda ${ }^{\circledR}, 1$ \\ ${ }^{1}$ Universidade Federal do Paraná \\ *paulomoreira@ufpr.br; ${ }^{\dagger} d t s u n o d a @ u f p r . b r$
}

Recebido: 28/02/2019. Revisado: 14/08/2019. Aceito: 10/09/2019.

\begin{abstract}
Resumo
Tem por objetivo classificar gêneros musicais automaticamente por meio de algoritmos de Mineração de Dados, considerando descritores extraídos do sinal de áudio. Identifica na Last.fm as 150 músicas mais populares de sete gêneros musicais (Rock, Jazz, POP, Música Clássica, MPB, Heavy Metal e Samba). Mediante a extração de descritores relacionados ao sinal de áudio destas músicas, aplica os algoritmos Random Forest; Bayes Net; C4.5; KNN e as estratégias Bagging e Boosting para a classificação. Obtém como melhor resultado 66,67\% de acerto com o algoritmo C4.5 para classificação entre Samba e MPB. Constata que a classificação de gêneros musicais se apresenta como um "problema interessante"para estudos que envolvem técnicas de Machine Learning. Estimula a continuidade de estudos semelhantes aplicando algoritmos baseados em Redes Neurais e Algoritmos Genéticos.
\end{abstract}

Palavras-Chave: Algoritmos; Classificação Automática; Gêneros Musicais; Mineração de Dados.

\section{Abstract}

It aims to classify musical genres automatically by means of Data Mining algorithms, considering descriptors extracted from the audio signal. It identifies the 150 most popular songs of seven musical genres (Rock, Jazz, POP, Classical Music, MPB, Heavy Metal and Samba). By extracting descriptors related to the audio signal of these songs, it applies algorithms Random Forest; Bayes Net; C4.5; KNN and the Bagging and Boosting strategies for the classification. It obtains the best result of $66.67 \%$ of success with the algorithm C4.5 for classification between Samba and MPB. It notes that the classification of musical genres presents itself as an "interesting problem"for studies involving Machine Learning techniques. It stimulates the continuity of similar studies applying algorithms based on Neural Networks and Genetic Algorithms.

Keywords: Algorithms; Automatic Classification; Musical Genres; Data Mining.

\section{Introdução}

Presente no dia a dia, a música é utilizada para uma gama de objetivos (Correa and Rodrigues, 2016), além de ser de grande importância, por exemplo, para atividades relacionadas à aprendizagem (Kotsifakos et al., 2013). Com o aumento considerável de bancos de dados musicais em número e tamanho nos últimos anos (Cor- rea and Rodrigues, 2016, Murphy and Koolagudi, 2018), e o avanço de tecnologias de computação móvel e de serviços de streaming de músicas (Spotify, Grooveshark, Pandora,...) (Wu and Jang, 2015, Cheng et al., 2017), percebe-se que a recuperação de música centrada no usuário tornou-se cada vez mais importante (Cheng et al., 2017).

Diante deste cenário, o desenvolvimento de abor- 
dagens para a recuperação de informações musicais (Music Information Retrieval (MIR)) transformou-se em um tópico importante dentro da Ciência da Computação (Correa and Rodrigues, 2016, Oramas et al., 2017).

Dentre as abordagens desenvolvidas, em particular, a classificação de gêneros musicais é uma das mais relevantes (Kotsifakos et al., 2013, Wu and Jang, 2015, Correa and Rodrigues, 2016), posto que, o gênero musical é uma das formas mais comuns no que tange ao gerenciamento da música digital (Nanni et al., 2016).

De acordo com Nanni et al. (2016), a classificação de gêneros musicais é uma tarefa crucial estudada pela comunidade de MIR desde 2002, contemplando diferentes descritores musicais e esquemas de classificação (Correa and Rodrigues, 2016).

A classificação automática de gêneros musicais desempenha um papel fundamental no contexto da indexação, da organização, da categorização e da recuperação de músicas (Correa and Rodrigues, 2016, Baniya and Lee, 2016). Desta forma, é de suma importância projetar abordagens eficientes para classificar gêneros musicais automaticamente (Baniya and Lee, 2016).

Diante deste contexto, o presente estudo objetiva classificar gêneros musicais automaticamente por meio de algoritmos de Mineração de Dados, considerando descritores extraídos do sinal de áudio.

A organização do presente artigo segue a seguinte divisão: a seção 2 descreve um conjunto de trabalhos correlatos ao tema apresentado. A seção 3 contempla os aspectos metodológicos adotados. A seção 4 corresponde às análises e à discussão dos resultados obtidos. Por fim, na seção 5, são apresentadas as considerações finais e as possibilidades de trabalhos futuros.

\section{Trabalhos Correlatos}

Dentre os primeiros trabalhos realizados com o objetivo de classificar automaticamente gêneros musicais, destaca-se os realizados por Deshpande et al. (2001) e Tzanetakis and Cook (2002). Deshpande et al. (2001) propuseram um sistema de classificação baseado na extração de 12 Coeficientes Cepstrais da Frequência Mel, somados a espectrogramas de um total de 157 músicas pertencentes aos gêneros Rock, Música Clássica e Jazz. Os autores optaram por utilizar apenas 20 segundos de cada registro e empregaram os seguintes algoritmos: a) K-Nearest Neighbour (KNN); b) Modelo Gaussiano; e c) Support Vector Machine (SVM).

Tzanetakis and Cook (2002) realizaram a classificação de gêneros musicais por meio de um conjunto de características relacionadas ao timbre, ao ritmo e à vibração do sinal de áudio. Neste trabalho, os autores desenvolveram uma base de dados denominada GTZAN, composta por segmentos de 30 segundos de 1000 músicas, de 10 diferentes gêneros: Blues, Música Clássica, Country, Disco, Hip Hop, Jazz, Heavy Metal, POP, Reggae e Rock.

Li et al. (2003) propuseram uma nova metodologia de extração de características baseada em informações obtidas por Daubechies Wavelet Coefficient Histograms (DWCHs). Para os experimentos, os métodos SVM e Linear
Discriminant Analysis (LDA) foram empregados em duas bases de dados: a) GTZAN; e b) uma base composta por 756 músicas, de cinco gêneros: Música Ambiente, Música Clássica, Fusion, Jazz e Rock.

Grimaldi et al. (2003) em seu trabalho utilizaram para o processo de extração de características a Discrete Wavelet Packet Transform (DWPT). Os autores aplicaram a DWPT em uma base de dados com 200 músicas, divididas em 5 gêneros musicais: Jazz, Música Clássica, Rock, Heavy Metal e Techno. Para a classificação, o algoritmo utilizado foi o KNN.

Huang et al. (2014) elaboraram um sistema automático de classificação de gêneros musicais baseado em uma estratégia de seleção de características locais, por meio da estratégia self-adaptive harmony search (SAHS). Os autores extraíram um conjunto de cinco características acústicas (intensidade, tom, timbre, tonalidade e ritmo) da base GTZAN e subtemeram estas ao classificador SVM.

Nanni et al. (2016) adotaram uma abordagem para a classificação de gêneros musicais baseada na fusão de diferentes conjuntos de recursos. Ao total, os autores utilizaram 11 diferentes descritores de textura extraídos da imagem do espectrograma e vários vetores de características acústicas. Em termos de experimentos, estes foram conduzidos em três bancos de dados: a) o Latin Music Database (LMD); b) o ISMIR 2004; e c) a base de dados GTZAN.

Costa et al. (2017) conduziram um experimento de classificação utilizando três bancos de dados com características distintas: a) uma coleção de músicas ocidentais denominada "ISMIR 2004 Database"; b) uma coleção de músicas latino-americanas denonimada "LMD Database;e, por fim, c) uma coleção de músicas africanas.

Quanto aos descritores, Costa et al. (2017) extraíram três conjuntos de descritores denominados como Rhythm Patterns (RP), Statistical Spectrum Descriptors (SSD) e Rhythm Histograms (RH). No tocante à tarefa de classificação, os autores propuseram uma comparação entre o algoritmo SVM e uma Rede Neural Convolucional.

Rosner and Kostek (2017) abordaram a classificação de gêneros musicais mediante a extração de características relacionadas ao áudio completo e aos instrumentos musicais específicos de cada gênero. O processo de extração de descritores ocorreu de duas formas: a) extração de características dos sinais originais; b) extração das características dos sinais separados para bateria (percussão), trompete, piano e saxofone.

Para os experimentos, Rosner and Kostek (2017) utilizaram mais de 8.000 trechos musicais representando 13 gêneros musicais, sendo estes:Rock Alternativo, Blues, Música Clássica, Country, Dance e DJ, Hard Rock e Metal, Jazz, Música Latina, New Age, POP, R\&B, Rap e Hip-Hop, Rock.

Tang et al. (2018) construíram uma estratégia Long Short Term Memory (LSTM) aplicada à classificação de gêneros musicais. Neste trabalho, os autores extraíram os Coeficientes Cepstrais da Frequência Mel dos arquivos presentes na base GTZAN, obtendo uma acurácia média de $50,00 \%$ para os 10 gêneros elencados nesta 
base de dados.

No cenário nacional, Romano and Adami (2015) propuseram um sistema de reconhecimento automático de gêneros musicais, explorando técnicas de extração por segmentos, sistemas multiexpert e a combinação de classificadores (paramétricos e não paramétricos). Os autores empregaram quatro grupos de descritores: a) timbre e Coeficientes Mel-Cepstrais; b) temporais; c) rítmicos; e d) pitch.

Como fonte de dados, os autores utilizaram duas bases: a) uma base experimental, elaborada a partir de um website de recomendações musicais; e b) a base de dados GTZAN. No tocante aos algoritmos de classificação, Romano and Adami (2015) fizeram o uso dos algoritmos Gaussian Mixture Model(GMM) e SVM. Como resultados, o estudo elaborado por Romano and Adami (2015) apresentou taxa de acerto de $46,20 \%$ para a base experimental e 75,10\% para a base GTZAN.

Após a exposição dos trabalhos relacionados ao tema deste estudo, as principais contribuições deste artigo consistem em:

- Construção e utilização de uma nova base de dados, cujo total de registros (1.050) é maior do que outras bases empregadas em estudos anteriores;

- Uso de gêneros musicais brasileiros (Samba e MPB) somados a gêneros tradicionais em tarefas de classificação de gêneros musicais;

- Comparação de diferentes algoritmos de classificação, empregando algoritmos com pouca aplicação em estudos semelhantes (C4.5; Random Forest) e classificadores bayesianos (Naive Bayes; Bayes Net);

- Utilização de diferentes descritores relacionados ao sinal de áudio.

\section{Materiais e Métodos}

Adotou-se para este estudo as etapas descritas no processo denominado Knowledge Discovery in Database (KDD) - Descoberta de Conhecimento em Bases de Dados - proposto por Fayyad et al. (1996). A seguir, descreve-se como cada uma das etapas deste processo foi realizada.

\subsection{Seleção}

Construiu-se uma base de dados composta por 1.050 registros com 25 atributos (24 representado descritores e um destinado ao gênero musical de cada registro) que representam as 150 músicas mais populares identificadas no dia 24 de junho de 2017 na plataforma Last.fm ${ }^{1}$ para os seguintes gêneros musicais: a) Rock (ROC), b) Jazz (JAZ), c) POP, d) Música Clássica (MC), e) MPB, f) Heavy Metal (HM); e g) Samba (SAM). Posterior à fase de identificação, extraiu-se o áudio de cada música diretamente de vídeos do Youtube por meio de um conversor online, respeitando a duração original destes.

\subsection{Pré-Processamento e Transformação}

Após a coleta dos áudios, realizou-se a transformação dos arquivos para o formato .wav, com frequência igual a $44100 \mathrm{~Hz}$ e taxa de bits de $1411 \mathrm{kbps}$, respectivamente. Devido à diferença de duração entre as músicas, padronizou-se a duração de cada arquivo para apenas 30 segundos, sendo estes correspondentes ao trigésimo segundo até o primeiro minuto de cada música.

Para o processo de extração dos 24 descritores utilizou-se a ferramenta open source jAudio ${ }^{2}$. A ferramenta em questão é desenvolvida em Java e permite a extração de 28 descritores relacionados ao sinal do áudio por meio de uma interface sem que haja a necessidade de executar linhas de códigos.

Neste estudo, utilizou-se os seguintes descritores:

i. Root Mean Square (RMS): representa a amplitude média quadrática de um sinal. É calculada a partir da Eq. (1):

$$
R M S=\sqrt{\frac{1}{2} \sum_{n=1}^{N} x[n]^{2}}
$$

ii. Taxa de Cruzamento Zero (Zero Crossings): informa quantas vezes o sinal cruza o eixo zero. Seu cálculo é realizado pela Eq. (2):

$$
\left.Z_{\mathrm{t}}=\frac{1}{2} \sum_{n=1}^{N} \mid \operatorname{sign}(x[n])-\operatorname{sign}(x[n-1])\right) \mid
$$

onde: 1 é utilizado para argumentos positivos; o para argumentos negativos; e $x[n]$ corresponde ao sinal no domínio do tempo para o frame [t].

iii. Centroide Espectral (Spectral Centroid): "é o ponto balanceado do espectro. É uma medida da forma espectral e é associado frequentemente com a noção do brilho espectral". (Silla Jr. et al., 2005). É calculado pela Eq. (3):

$$
S C_{t}=\frac{\sum_{n=1}^{N} P_{t}[n] * n}{\sum_{n=1}^{N} P_{t}[n]}
$$

onde: $P_{t}[n]$ representa a magnitude da transformada de Fourier no frame $t$ na frequência $n$.

iv. Rolloff Espectral (Spectral Rolloff): medida espectral definida como a frequência $\mathrm{SR}_{\mathrm{t}}$, na qual $\mathrm{k}$ da magnitude da distribuição está concentrada (Silla Jr. et al., 2005). Para k, normalmente, adota-se os valores de 0,85 ou 0,95 (McKay, 2010). Este descritor é obtido por meio da Eq. (4):

$$
\sum_{n=1}^{S R_{t}} P_{t}[n]=k \sum_{n=1}^{N} P_{t}[n]
$$

Neste estudo, adotou-se 0,85 para o valor de $\mathrm{k}$. v. Fluxo Espectral (Spectral Flux): medida relacionada à mudança espectral de um sinal (McKay, 2010). É obtida pela Eq. (5):

\footnotetext{
${ }^{1}$ https://www.last.fm/pt/home
}

\footnotetext{
$2 \overline{\mathrm{http}: / / \text { jaudio.sourceforge.net/ }}$
} 


$$
S F_{t}=\sum_{n=1}^{n}\left(N_{t}[n]-N_{t-1}[n]\right)^{2}
$$

onde: $N_{t}[n]$ e $N_{t-1}[n]$ correspondem à magnitude normalizada da transformada de Fourier no frame atual $t$ e no frame anterior $t-1$, respectivamente.

vi. Compactness: descritor baseado no trabalho de Mcadams (1999), obtido, geralmente, com base no espectro de magnitude (McKay, 2010).

vii. Baixa Energia (Fraction of Low Energy Frames): obtida por meio da análise de um conjunto de janelas consecutivas que têm um valor RMS abaixo de algum limite (McKay, 2010).

viii. Coeficientes Cepstrais da frequência Mel (Melfrequency cepstral coefficients (MFCC)): "são características perceptualmente motivadas que também são baseadas na transformada de Fourier (Short Time Fourier Transform (STFT))" (Silla Jr. et al., 2005). Normalmente, utiliza-se 13 coeficientes desta escala (McKay, 2010). Contudo, estudos indicam que cinco coeficientes apresentam resultados melhores para a classificação de gêneros musicais (Tzanetakis and Cook, 2002, Silla Jr. et al., 2005.)

Para a extração de cada descritor, trabalhou-se com os valores referentes à média e ao desvio padrão dos descritores i a vii, além da média e o desvio padrão dos cinco primeiros coeficientes do descritor de número viii. Em todo o processo de extração utilizou-se uma janela de 512 amostras, sem sobreposição.

\subsection{Mineração de Dados}

Os procedimentos de Mineração de Dados adotados foram realizados na ferramenta Weka $3.8^{3}$. No que tange aos algoritmos de classificação, empregou-se os seguintes algoritmos: a) Random Forest (RF) (Breiman, 2001); b) Bayes Net (BN) (Friedman et al., 1997); c) KNN (Cover and Hart, 1967); d) C4.5 (Quinlan, 1993); e e) Naive Bayes (NB) (Friedman et al., 1997). Por fim, combinou-se cada um destes algoritmos com as estratégias Bagging (Breiman, 1996) e Boosting (Freund and Schapire, 1997).

Em todos os experimentos adotou-se como padrão o uso de uma validação cruzada (cross-validation) (Stone, 1974) com dez partições.

\section{Análise e Discussão dos Resultados}

Ao aplicar-se os algoritmos de classificação, percebeuse que a taxa de acerto obtida para os gếneros Samba e MPB nunca ultrapassou os 50,00\% de acerto em nenhum dos cenários com a presença dos sete gêneros. Desta forma, optou-se pela adoção de três estratégias de classificação: a) com os sete gêneros; b) com cinco gêneros, retirando os gêneros Samba e MPB; e c) classificação binária apenas entre os gêneros Samba e MPB.

\footnotetext{
3 https://www.cs.waikato.ac.nz/ml/weka/
}

\subsection{Resultados obtidos com o algoritmo Ran- dom Forest}

Para o algoritmo Random Forest, variou-se o parâmetro I que corresponde ao número de iterações. Para este parâmetro adotou-se valores de $\mathrm{I}=100$ ( default) a $\mathrm{I}=1000$. Os resultados para este algoritmo são representados na Tabela 1.

Tabela 1: Resultados Random Forest

\begin{tabular}{cccc}
\hline Iterações & 7 gêneros & 5 gêneros & 2 gêneros \\
\hline 100 & $47,53 \%$ & $61,20 \%$ & $57,00 \%$ \\
200 & $47,90 \%$ & $62,13 \%$ & $57,33 \%$ \\
300 & $48,10 \%$ & $61,60 \%$ & $57,00 \%$ \\
400 & $49,05 \%$ & $62,00 \%$ & $56,33 \%$ \\
500 & $48,38 \%$ & $62,53 \%$ & $57,33 \%$ \\
600 & $48,20 \%$ & $62,40 \%$ & $56,33 \%$ \\
700 & $48,10 \%$ & $62,13 \%$ & $56,67 \%$ \\
800 & $47,52 \%$ & $62,40 \%$ & $56,00 \%$ \\
900 & $47,62 \%$ & $62,27 \%$ & $56,33 \%$ \\
1000 & $47,52 \%$ & $62,00 \%$ & $56,33 \%$ \\
\hline
\end{tabular}

Fonte: Elaborado pelos Autores (2019).

Verifica-se na Tabela 1 que, para a classificação com sete gêneros, não obteve-se nenhum cenário com taxa de acerto superior a 50,00\%, sendo o maior valor obtido ao adotar $\mathrm{I}=400(49,05 \%)$.

Por sua vez, ao utilizar-se apenas cinco gêneros, percebe-se resultados superiores para todos os valores de I, com todos os resultados com taxa de acerto acima de $60,00 \%$, sendo o maior valor obtido para $\mathrm{I}=500(62,53 \%)$. No tocante à matriz de confusão para o melhor resultado obtido para a classificação considerando sete gêneros, esta é representada pela Tabela 2.

Tabela 2: Matriz de Confusão Random Forest (7 gêneros)

\begin{tabular}{cccccccc}
\hline & SAM & ROC & POP & MPB & JAZ & HM & MC \\
\hline SAM & 49 & 12 & 17 & 44 & 23 & 2 & 3 \\
ROC & 11 & 53 & 20 & 11 & 11 & 31 & 13 \\
POP & 22 & 14 & 70 & 18 & 13 & 10 & 3 \\
MPB & 44 & 11 & 13 & 53 & 24 & 2 & 3 \\
JAZ & 20 & 12 & 10 & 20 & 69 & 4 & 15 \\
HM & 2 & 27 & 10 & 7 & 7 & 90 & 6 \\
MC & 2 & 4 & 0 & 1 & 12 & 0 & 131 \\
\hline \multicolumn{7}{c}{ Fonte: Elaborado pelos Autores (2019). }
\end{tabular}

Com base na análise da Tabela 2 , destaca-se o desempenho para a classificação dos gêneros Música Clássica (MC) com 87,33\% de acerto, e Heavy Metal (HM), com $60,00 \%$ de instâncias classificadas corretamente. Quanto ao menor desempenho, o gênero Samba apresentou uma taxa de apenas $32,67 \%$ de instâncias classificadas corretamente.

Com relação ao desempenho para a base com cinco gêneros, com base nos resultados elencados na Tabela 1, verifica-se que, para todas as variações adotadas, a taxa 
de acerto foi superior a $60,00 \%$, sendo que, ao considerar o número de 500 iterações obteve-se um total de $62,53 \%$ de acerto. A matriz de confusão para os resultados da classificação com cinco gêneros é representada na Tabela 3.

Tabela 3: Matriz de confusão Random Forest (5 gêneros)

\begin{tabular}{cccccc}
\hline & ROC & POP & JAZ & HM & MC \\
\hline ROC & $\mathbf{6 1}$ & $\mathbf{2 9}$ & 19 & 30 & 11 \\
POP & 22 & $\mathbf{9 4}$ & 22 & 9 & 3 \\
JAZ & 17 & 18 & $\mathbf{9 8}$ & 2 & 15 \\
HM & 33 & 11 & 13 & $\mathbf{8 6}$ & 7 \\
MC & 6 & 0 & 13 & 1 & $\mathbf{1 3 0}$ \\
\hline
\end{tabular}

Fonte: Elaborado pelos Autores (2019).

Analisando a Tabela 3, destaca-se que três gêneros apresentaram uma taxa de acerto superior a 60,00\%: Música Clássica (86,67\%), Jazz (65,33\%) e POP $(62,67 \%)$. Por sua vez, o gênero Rock apresentou uma taxa de acerto de 40,67\%, a menor dentre os cinco gêneros.

No tocante à classificação binária entre Samba e MPB, o melhor resultado em termos de classificação foi obtido mediante o uso de 200 e 500 iterações; ambos os cenários apresentaram uma taxa de acerto de 57,33\%. A matriz de confusão para a classificação entre Samba e MPB é representada na Tabela 4.

Tabela 4: Matriz de confusão Random Forest (2 gêneros)

\begin{tabular}{ccc}
\hline & SAM & MPB \\
\hline SAM & $\mathbf{8 6}$ & 64 \\
MPB & 64 & $\mathbf{8 6}$ \\
\hline
\end{tabular}

Fonte: Elaborado pelos Autores (2019).

Verifica-se na Tabela 4, que o desempenho para os dois gêneros foi o mesmo $(57,00 \%)$ o que reforça a dificuldade de distinção entre estes dois gêneros.

\subsection{Resultados obtidos com o algoritmo Bayes Net}

Para o algoritmo Bayes Net, a variação de parâmetros ocorreu com os parâmetros relacionados à estimação das probabilidades (estimator) e ao método de busca (searchAlgorithm). Os resultados são elencados na Tabela 5 .

Observando-se a Tabela 5 nota-se que, não houve cenários com taxa de acerto superior a $50,00 \%$ no que tange à classificação considerando sete gêneros musicais. Nesta classificação,o maior valor $(45,43 \%)$ corresponde à combinação entre o método de estimação SimpleEstimator e o algoritmo de busca Tan.

No tocante à matriz de confusão para o melhor resultado obtido para a classificação considerando sete gêneros, esta é representada pela Tabela 6 .

Com base na análise da Tabela 6, destaca-se que
Tabela 5: Resultados Bayes Net

\begin{tabular}{ccc}
\hline Parâmetros & 7 gêneros & 5 gêneros \\
\hline Default (SE e K2) & $45,05 \%$ & $58,93 \%$ \\
SE e Tan & $45,43 \%$ & $59,47 \%$ \\
SE e TabuSearch & $44,86 \%$ & $45,05 \%$ \\
SE e HillClim. & $45,05 \%$ & $58,93 \%$ \\
SE e LAGDHillClim. & $44,30 \%$ & $58,80 \%$ \\
SE e RepeatedHillClim. & $45,05 \%$ & $58,93 \%$ \\
BMAEst. e K2 & $43,81 \%$ & $58,93 \%$ \\
BMAEst. e HillClim. & $43,81 \%$ & $58,93 \%$ \\
BMAEst. e RepeatedHillClim. & $43,81 \%$ & $58,93 \%$ \\
\hline Fonte: Elaborado pelos Autores (2019).
\end{tabular}

Tabela 6: Matriz de confusão Bayes Net (7 gêneros)

\begin{tabular}{cccccccc}
\hline & SAM & ROC & POP & MPB & JAZ & HM & MC \\
\hline SAM & 54 & 12 & 20 & 28 & 30 & 3 & 3 \\
ROC & 14 & $\mathbf{4 2}$ & 20 & 16 & 15 & 32 & 11 \\
POP & 23 & 20 & 64 & 19 & 11 & 11 & 2 \\
MPB & 36 & 19 & 18 & $\mathbf{4 3}$ & 28 & 3 & 3 \\
JAZ & 21 & 13 & 14 & 24 & $\mathbf{6 2}$ & 3 & 13 \\
HM & 7 & 27 & 5 & 12 & 6 & $\mathbf{8 8}$ & 5 \\
MC & 0 & 9 & 0 & 5 & 10 & 2 & $\mathbf{1 2 4}$
\end{tabular}

Fonte: Elaborado pelos Autores (2019).

apenas os gêneros Música Clássica (82,67\%) e Heavy Metal $(58,67 \%)$ apresentaram taxa de instâncias classificadas corretamente acima dos 50,00\%. Quanto ao menor desempenho, o gênero Rock teve apenas $28,00 \%$ de instâncias classificadas corretamente.

Quanto à classificação com cinco gêneros, percebese um aumento no desempenho, com apenas a combinação entre o método de estimação SimpleEstimator e o algoritmo de busca TabuSearch com resultado inferior a $50,00 \%$. Considerando cinco gêneros musicais, a combinação entre o método de estimação SimpleEstimator e o algoritmo de busca Tan resultou no maior valor obtido em termos de classificação (59,47\%). A matriz de confusão para o melhor resultado obtido neste cenário é representada pela Tabela 7 .

Tabela 7: Matriz de confusão Bayes Net (5 gêneros)

\begin{tabular}{cccccc}
\hline & ROC & POP & JAZ & HM & MC \\
\hline ROC & 54 & 31 & 20 & 34 & 11 \\
POP & 28 & $\mathbf{8 4}$ & 23 & 13 & 2 \\
JAZ & 19 & 19 & $\mathbf{9 0}$ & 7 & 15 \\
HM & 34 & 14 & 9 & $\mathbf{8 8}$ & 5 \\
MC & 10 & 1 & 8 & 1 & $\mathbf{1 3 0}$ \\
\hline Fonte: Elaborado pelos Autores (2019).
\end{tabular}

Com base na análise da Tabela 7, destaca-se os $86,67 \%$ de acerto para o gênero Música Clássica. Os demais gêneros apresentaram taxa de acerto superior a 50,00\%, com exceção ao gênero Rock, cuja taxa foi de apenas $36,00 \%$ de instâncias classificadas corretamente.

Quanta à classificação binária entre Samba e MPB, todos os cenários obtiveram $60,67 \%$ de taxa de acerto. A matriz de confusão para a classificação entre Samba e MPB é representada na Tabela 8 . 
Tabela 8: Matriz de confusão Bayes Net (2 gêneros)

\begin{tabular}{ccc}
\hline & SAM & MPB \\
\hline SAM & $\mathbf{9 6}$ & 54 \\
MPB & 64 & $\mathbf{8 6}$
\end{tabular}

Fonte: Elaborado pelos Autores (2019).

Verifica-se na Tabela 8, que o desempenho para os dois gêneros foi superior a 50,00\% de taxa de acerto, sendo $64,00 \%$ para o gênero Samba e $57,00 \%$ para a MPB.

\subsection{Resultados obtidos com o algoritmo KNN}

Para o algoritmo KNN, variou-se o parâmetro K que corresponde ao número de vizinhos utilizados para a classificação. Para este parâmetro adotou-se valores de $\mathrm{K}=1$ ( default) a $\mathrm{K}=15$. Os resultados para este algoritmo são representados na Tabela 9.

Tabela 9: Resultados KNN

\begin{tabular}{cccc}
\hline $\mathbf{K}$ & $\mathbf{7}$ gêneros & 5 gêneros & 2 gêneros \\
\hline $\mathbf{1}$ & $31,71 \%$ & $43,33 \%$ & $41,00 \%$ \\
3 & $32,48 \%$ & $45,33 \%$ & $50,33 \%$ \\
5 & $33,24 \%$ & $48,27 \%$ & $51,67 \%$ \\
7 & $34,86 \%$ & $50,57 \%$ & $51,67 \%$ \\
9 & $37,33 \%$ & $49,60 \%$ & $53,00 \%$ \\
11 & $36,95 \%$ & $49,47 \%$ & $53,67 \%$ \\
13 & $38,10 \%$ & $51,60 \%$ & $57,33 \%$ \\
15 & $38,48 \%$ & $51,20 \%$ & $54,33 \%$ \\
\hline
\end{tabular}

Fonte: Elaborado pelos Autores (2019).

Observa-se na Tabela 9 que, para a classificação com sete gêneros, nenhum cenário apresentou taxa de acerto superior a $40,00 \%$, sendo o maior valor obtido ao adotar-se um total de 15 vizinhos $(38,48 \%)$.

No tocante à matriz de confusão para o melhor resultado considerando sete gêneros, esta é representada pela Tabela 10.

Tabela 10: Matriz de confusão KNN (7 gêneros)

\begin{tabular}{cccccccc}
\hline & SAM & ROC & POP & MPB & JAZ & HM & MC \\
\hline SAM & $\mathbf{6 9}$ & 13 & 14 & 20 & 17 & 11 & 6 \\
ROC & 31 & $\mathbf{4 5}$ & 11 & 17 & 6 & 26 & 14 \\
POP & 44 & 25 & $\mathbf{4 1}$ & 14 & 10 & 13 & 3 \\
MPB & 60 & 15 & 11 & 34 & 20 & 6 & 4 \\
JAZ & 52 & 15 & 11 & 9 & 34 & 6 & 15 \\
HM & 18 & 40 & 10 & 7 & 4 & $\mathbf{6 1}$ & 10 \\
MC & 7 & 8 & 0 & 3 & 7 & 5 & $\mathbf{1 2 0}$ \\
\hline
\end{tabular}

Fonte: Elaborado pelos Autores (2019).

Com base na análise da Tabela 10, verifica-se que apenas houve taxa de acerto superior a $50,00 \%$ para o gênero Música Clássica $(80,00 \%)$. Os gêneros Jazz e MPB apresentaram os menores resultados: apenas $22,67 \%$ dos registros destes gêneros foram classificados corretamente.
Ao utilizar-se apenas cinco gêneros, percebe-se resultados superiores para todos os valores de $\mathrm{K}$, com três resultados com taxa de acerto acima de 50,00\%: a) $\mathrm{K}=7(50,67 \%)$; b) $\mathrm{K}=13$ (51,60\%); e c) $\mathrm{K}=15(51,20 \%)$. A matriz de confusão para os resultados da classificação com cinco gêneros é representada na Tabela 11.

Tabela 11: Matriz de confusão KNN (5 gêneros)

\begin{tabular}{cccccc}
\hline & ROC & POP & JAZ & HM & MC \\
\hline ROC & $\mathbf{6 7}$ & 24 & 15 & 30 & 14 \\
POP & 44 & 65 & 18 & 20 & 3 \\
JAZ & 33 & 19 & $\mathbf{7 0}$ & 8 & 20 \\
HM & 57 & 16 & 7 & $\mathbf{6 1}$ & 9 \\
MC & 11 & 2 & 8 & 5 & $\mathbf{1 2 4}$ \\
\hline Fonte: Elaborado pelos Autores (2019).
\end{tabular}

Analisando a Tabela 11, destaca-se que, assim como a classificação considerando os sete gêneros, apenas a Música Clássica $(82,67 \%)$ apresentou mais do que $50,00 \%$ de instâncias classificadas corretamente.

No que tange à classificação entre Samba e MPB, o melhor resultado foi obtido mediante a adoção de $\mathrm{K}=13$. Neste cenário, obteve-se uma taxa de acerto de 57,33\%. A matriz de confusão para a classificação entre Samba e MPB é representada na Tabela 12.

Tabela 12: Matriz de confusão KNN (2 gêneros)

\begin{tabular}{ccc}
\hline & SAM & MPB \\
\hline SAM & 104 & 46 \\
MPB & 82 & 68 \\
\hline
\end{tabular}

Fonte: Elaborado pelos Autores (2019).

Percebe-se na Tabela 12, que o desempenho para o gênero Samba $(69,33 \%)$ foi superior à classificação para o gênero MPB $(45,33 \%)$.

\subsection{Resultados obtidos com o algoritmo C4.5}

Com relação ao algoritmo C4.5 -implementado na ferramenta Weka como J48 - adotou-se variações para os parâmetros de confiança (confidenceFactor (C)) e o número mínimo de instâncias por folha (minNumObj $(\mathrm{mNO})$ ). Os resultados para este algoritmo são representados na Tabela 13.

Tabela 13: Resultados C4.5

\begin{tabular}{cccc}
\hline Parâmetros & 7 gêneros & 5 gêneros & 2 gêneros \\
\hline Default & $37,71 \%$ & $51,33 \%$ & $59,67 \%$ \\
$\mathrm{C}(0,1)$ & $38,57 \%$ & $51,47 \%$ & $60,67 \%$ \\
$\mathrm{C}(0,1) ; \mathrm{mNO}(5)$ & $39,04 \%$ & $51,47 \%$ & $62,00 \%$ \\
$\mathrm{C}(0,1) ; \mathrm{mNO}(20)$ & $40,23 \%$ & $51,07 \%$ & $66,00 \%$ \\
$\mathrm{C}(0,1) ; \mathrm{mNO}(30)$ & $40,86 \%$ & $52,13 \%$ & $66,67 \%$ \\
\hline
\end{tabular}

Fonte: Elaborado pelos Autores (2019).

Observando-se a Tabela 13 verifica-se que, para a classificação com sete gêneros, nenhum cenário apresentou taxa de acerto superior a $45,00 \%$, sendo o maior 
valor obtido ao adotar-se o valor de 0,1 para a confiança (confidenceFactor) e um número de instâncias (minNumObj) igual a 30. O valor para estas combinações resultou em 40,86\% de acerto.

No tocante à matriz de confusão para o melhor resultado considerando sete gêneros, esta é representada pela Tabela 14 .

Tabela 14: Matriz de confusão C4.5 (7 gêneros)

\begin{tabular}{cccccccc}
\hline & SAM & ROC & POP & MPB & JAZ & HM & MC \\
\hline SAM & $\mathbf{4 1}$ & 16 & 14 & 31 & 30 & 13 & 5 \\
ROC & 13 & 38 & 21 & 12 & 23 & 34 & 9 \\
POP & 17 & 20 & 45 & 18 & 19 & 23 & 8 \\
MPB & 29 & 17 & 8 & $\mathbf{4 9}$ & 29 & 10 & 8 \\
JAZ & 15 & 14 & 8 & 27 & 65 & 6 & 15 \\
HM & 9 & 19 & 15 & 9 & 9 & $\mathbf{8 2}$ & 7 \\
MC & 1 & 13 & 2 & 5 & 18 & 2 & $\mathbf{1 0 9}$ \\
\hline
\end{tabular}

Fonte: Elaborado pelos Autores (2019).

Com base na análise da Tabela 14 , verifica-se que apenas houve taxa de acerto superior a $50,00 \%$ para os gêneros Música Clássica (72,67\%) e Heavy Metal $(54,67 \%)$. O pior resultado neste cenário foi para o gênero Rock com apenas $25,33 \%$ dos registros classificados corretamente.

Ao utilizar-se cinco gêneros, percebe-se resultados superiores a $50,00 \%$ para todas as variações. Com cinco gêneros obteve-se como melhor resultado o valor de $52,13 \%$ de acerto ao utilizar-se uma confiança de 0,1 e o número de instâncias igual a 30. A matriz de confusão para o melhor resultado obtido considerando cinco gêneros é representada na Tabela 15.

Tabela 15: Matriz de confusão C4.5 (5 gêneros)

\begin{tabular}{cccccc}
\hline & ROC & POP & JAZ & HM & MC \\
\hline ROC & $\mathbf{4 1}$ & 33 & $\mathbf{2 1}$ & 33 & $\mathbf{2 2}$ \\
POP & $\mathbf{2 5}$ & $\mathbf{7 2}$ & $\mathbf{2 9}$ & 18 & 6 \\
JAZ & 17 & $\mathbf{2 2}$ & $\mathbf{8 0}$ & 10 & $\mathbf{2 1}$ \\
HM & $\mathbf{2 5}$ & $\mathbf{1 4}$ & $\mathbf{1 7}$ & $\mathbf{8 6}$ & 8 \\
MC & $\mathbf{1 2}$ & 7 & $\mathbf{1 9}$ & $\mathbf{0}$ & $\mathbf{1 1 2}$ \\
\hline
\end{tabular}

Fonte: Elaborado pelos Autores (2019).

Analisando a Tabela 15, destaca-se o desempenho para a classificação dos gêneros Música Clássica (74,67\%), Heavy Metal (57,33\%) e Jazz (53,33\%).

No que tange à classificação entre Samba e MPB, o melhor resultado correspondeu a $66,67 \%$ de acerto, mediante a adoção dos mesmos parâmetros que resultaram no melhor cenário para a classificação das estratégias com sete e cinco gêneros. A matriz de confusão para a classificação entre Samba e MPB é representada na Tabela 16.

Tabela 16: Matriz de confusão C4.5 (2 gêneros)

\begin{tabular}{ccc}
\hline & SAM & MPB \\
\hline SAM & $\mathbf{9 4}$ & 66 \\
MPB & 44 & $\mathbf{1 0 6}$ \\
\hline
\end{tabular}

Fonte: Elaborado pelos Autores (2019).
Percebe-se na Tabela 16 um desempenho satisfatório para ambos os gêneros, posto que, obteve-se $62,67 \%$ de acerto para o gênero Samba e 70,67\% de instâncias classificadas corretamente para o gênero MPB.

\subsection{Resultados obtidos com o algoritmo Naive Bayes}

Para o algoritmo Naive Bayes, adotou-se três cenários de classificação: a) parâmetros default; b) parâmetro useKernelEstimator (True); e c) parâmetro useSupervisedDiscretization (True). Os resultados obtidos são listados na Tabela 17.

Tabela 17: Resultados Naive Bayes

\begin{tabular}{lccc}
\hline \multicolumn{1}{c}{ Parâmetros } & 7 gêneros & 5 gêneros & 2 gêneros \\
\hline Default & $44,10 \%$ & $57,87 \%$ & $54,67 \%$ \\
useKernelEst. & $46,95 \%$ & $59,67 \%$ & $56,33 \%$ \\
useSupervisedDis. & $45,14 \%$ & $59,20 \%$ & $60,67 \%$ \\
\hline
\end{tabular}

Fonte: Elaborado pelos Autores (2019).

Para os cenários considerando os sete gêneros, ao observar-se a Tabela 17 verifica-se que o melhor cenário consistiu em 46,95\% de instâncias classificadas corretamente. A matriz de confusão para o resultado deste cenário é representada pela Tabela 18.

Tabela 18: Matriz de confusão Naive Bayes (7 gêneros)

\begin{tabular}{cccccccc}
\hline & SAM & ROC & POP & MPB & JAZ & HM & MC \\
\hline SAM & 49 & 5 & 20 & 49 & 17 & 5 & 5 \\
ROC & 9 & 29 & 24 & 25 & 10 & 40 & 13 \\
POP & 14 & 8 & 73 & 25 & 11 & 15 & 4 \\
MPB & 34 & 3 & 14 & 64 & 31 & 3 & 1 \\
JAZ & 26 & 9 & 12 & 28 & 55 & 4 & 16 \\
HM & 7 & 17 & 10 & 7 & 7 & 95 & 7 \\
MC & 1 & 6 & 0 & 4 & 5 & 6 & $\mathbf{1 2 8}$ \\
\hline \multicolumn{5}{c}{ Fonte: Elaborado pelos Autores (2019). }
\end{tabular}

Com base na Tabela 18, nota-se que, somente os gêneros Música Clássica (85,33\%) e Heavy Metal (63,33\%) superaram os $50,00 \%$ de acerto. Por sua vez, o gênero Rock apresentou uma taxa de acerto de apenas 19,33\%, sendo o gênero com o pior desempenho em termos de classificação.

Com relação à classificação com cinco gêneros, os três cenários realizados tiveram valores superiores a $55,00 \%$ de instâncias classificadas corretamente, sendo o maior valor obtido ao utilizar-se o parâmetro useKernelEstimator igual a True $(59,67 \%)$. A matriz de confusão para o melhor cenário com cinco gêneros é apresentada na Tabela 19.

Com relação à interpretação dos resultados demonstrados na Tabela 19, percebe-se que apenas o gênero Rock $(25,33 \%)$ não obteve desempenho superior a 50,00\%. Com relação aos demais gêneros, a Música Clássica foi o gênero com o maior número de instâncias corretamente classificadas $(86,00 \%)$.

Para a classificação binária entre os gêneros Samba e MPB, ao adotar-se o parâmetro useSupervisedDiscre- 
Tabela 19: Matriz de confusão Naive Bayes ( 5 gêneros)

\begin{tabular}{cccccc}
\hline & ROC & POP & JAZ & HM & MC \\
\hline ROC & $\mathbf{3 8}$ & 35 & $\mathbf{2 0}$ & 44 & 13 \\
POP & 11 & $\mathbf{9 2}$ & 27 & 17 & 3 \\
JAZ & 14 & 21 & $\mathbf{8 9}$ & 8 & 18 \\
HM & 27 & 10 & 11 & $\mathbf{9 5}$ & 7 \\
MC & 7 & 0 & 8 & 6 & $\mathbf{1 2 9}$ \\
\hline
\end{tabular}

Fonte: Elaborado pelos Autores (2019).

tization igual a True, obteve-se uma taxa de acerto de $60,67 \%$. Os resultados deste cenário são listados na Tabela 20.

Tabela 20: Matriz de confusão Naive Bayes (2 gêneros)

\begin{tabular}{ccc}
\hline & SAM & MPB \\
\hline SAM & 96 & 54 \\
MPB & 64 & $\mathbf{8 6}$ \\
\hline
\end{tabular}

Fonte: Elaborado pelos Autores (2019).

Na matriz de confusão descrita na Tabela 20 , verificase $64,00 \%$ de acerto para o gênero Samba e $57,33 \%$ de acerto para a MPB.

\subsection{Resultados obtidos com a estratégia Bag- ging}

Para realizar a classificação mediante o uso da estratégia Bagging, combinou-se os melhores resultados obtidos por cada um dos algoritmos utilizados anteriormente com os parâmetros default do algoritmo Bagging. Os resultados obtidos são representados na Tabela 21.

Tabela 21: Resultados Bagging

\begin{tabular}{cccc}
\hline Algoritmo & 7 gêneros & 5 gêneros & 2 gêneros \\
\hline RF & $48,38 \%$ & $62,67 \%$ & $58,33 \%$ \\
BN & $50,85 \%$ & $62,40 \%$ & $61,67 \%$ \\
KNN & $39,05 \%$ & $51,47 \%$ & $55,33 \%$ \\
C4.5 & $45,52 \%$ & $57,47 \%$ & $62,00 \%$ \\
NB & $46,47 \%$ & $58,48 \%$ & $61,67 \%$ \\
\hline
\end{tabular}

Fonte: Elaborado pelos Autores (2019).

Os resultados elencados na Tabela 21 foram obtidos mediante os seguintes cenários: a) Rando Forest com 500 iterações (I(500)); b) Bayes Net (SimpleEstimator e TAN); c) KNN com o valor de K(13); d) C4.5 com confiança igual a 0,1 e número mínimo de instâncias igual a 30; e e) Naive Bayes com o parâmetro useSupervisedDiscretization igual a True.

Com base na Tabela 21, observa-se que, para a classificação considerando os sete gêneros, o único cenário com taxa de acerto superior a $50,00 \%$ correspondeu à combinação entre a estratégia Bagging e o algoritmo Bayes Net, considerando o método de estimação "SimpleEstimator"e o algoritmo de busca "Tan". A matriz de confusão para estes resultados é elencada na Tabela 22 .

Conforme verifica-se na Tabela 22, os gêneros Mú-
Tabela 22: Matriz de confusão Bagging (7 gêneros)

\begin{tabular}{cccccccc}
\hline & SAM & ROC & POP & MPB & JAZ & HM & MC \\
\hline SAM & $\mathbf{5 9}$ & $\mathbf{1 5}$ & $\mathbf{1 7}$ & 33 & $\mathbf{2 1}$ & 5 & 0 \\
ROC & 11 & $\mathbf{5 2}$ & 23 & $\mathbf{1 3}$ & $\mathbf{1 2}$ & 31 & 8 \\
POP & $\mathbf{1 8}$ & $\mathbf{2 2}$ & $\mathbf{7 1}$ & $\mathbf{2 1}$ & $\mathbf{1 0}$ & 6 & 2 \\
MPB & 35 & 16 & $\mathbf{1 8}$ & $\mathbf{5 2}$ & $\mathbf{2 5}$ & 2 & 2 \\
JAZ & $\mathbf{1 5}$ & 9 & 14 & $\mathbf{2 1}$ & $\mathbf{7 5}$ & $\mathbf{4}$ & $\mathbf{1 2}$ \\
HM & 3 & 34 & 6 & 5 & 5 & $\mathbf{9 2}$ & 5 \\
MC & 0 & 1 & 2 & 2 & 9 & 3 & $\mathbf{1 3 3}$ \\
\hline
\end{tabular}

Fonte: Elaborado pelos Autores (2019).

sica Clássica $(88,67 \%)$ e Heavy Metal $(61,33 \%)$ foram os únicos com taxa de acerto superior a $50,00 \%$; neste cenário, o gênero Jazz teve exatamente $50,00 \%$ dos seus registros classificados corretamente.

Com relação à classificação com cinco gêneros, observa-se na Tabela 21 que o melhor resultado em termos de acerto foi obtido ao utilizar-se o algoritmo Random Forest com o valor de 500 iterações $(62,67 \%)$. Este cenário é representado na Tabela 23.

Tabela 23: Matriz de confusão Bagging (5 gêneros)

\begin{tabular}{cccccc}
\hline & ROC & POP & JAZ & HM & MC \\
\hline ROC & 57 & 29 & 23 & 31 & 10 \\
POP & 24 & $\mathbf{9 0}$ & 23 & 10 & 3 \\
JAZ & 14 & 15 & $\mathbf{1 0 3}$ & 3 & 15 \\
HM & 34 & 10 & 12 & $\mathbf{8 8}$ & 6 \\
MC & 5 & 0 & 12 & 1 & 132 \\
\hline
\end{tabular}

Fonte: Elaborado pelos Autores (2019).

Conforme exposto na Tabela 23, destaca-se os resultados obtidos para os gêneros Música Clássica $(88,67 \%)$ e Jazz $(68,67 \%)$. Dos cinco gêneros, neste cenário, apenas o gênero Rock $(38,00 \%)$ ficou abaixo dos $50,00 \%$ de acerto.

Quanto à classificação considerando apenas os gêneros Samba e MPB, o melhor resultado consistiu na combinação do algoritmo Bagging com o algoritmo C4.5, utilizando confiança de 0,1 e considerando o número mínimo de instâncias igual a 30. Esta combinação resultou em $62,00 \%$ de instâncias corretamente classificadas. Os resultados deste cenário são elencados na Tabela 24.

Tabela 24: Matriz de confusão Bagging (2 gêneros)

\begin{tabular}{ccc} 
& SAM & MPB \\
\hline SAM & $\mathbf{9 8}$ & 52 \\
MPB & 62 & $\mathbf{8 8}$ \\
\hline
\end{tabular}

Fonte: Elaborado pelos Autores (2019).

Observando-se a Tabela 24, tem-se uma taxa de acerto de $65,00 \%$ para o gênero Samba e 59,00\% de instâncias classificadas corretamente para o gênero MPB. 


\subsection{Resultados obtidos com a estratégia Boos- ting}

Para realizar a classificação mediante o uso da estratégia Boosting, combinou-se as estratégias adotadas para cada um dos algoritmos utilizados anteriormente com os parâmetros default do algoritmo AdaBoostM1.

Os melhores resultados para cada algoritmo são representados na Tabela 25.

Tabela 25: Resultados Boosting

\begin{tabular}{cccc}
\hline Algoritmo & 7 gêneros & 5 gêneros & 2 gêneros \\
\hline RF & $48,00 \%$ & $62,40 \%$ & $58,33 \%$ \\
BN & $45,42 \%$ & $59,47 \%$ & $60,67 \%$ \\
KNN & $38,10 \%$ & $51,60 \%$ & $57,33 \%$ \\
C4.5 & $40,85 \%$ & $55,20 \%$ & $65,00 \%$ \\
NB & $46,95 \%$ & $59,07 \%$ & $51,33 \%$ \\
\hline
\end{tabular}

Fonte: Elaborado pelos Autores (2019).

Os resultados elencados na Tabela 25 foram obtidos mediante os seguintes cenários: a) Rando Forest com 100 iterações (I(100)); b) Bayes Net (SimpleEstimator e TAN); c) KNN com o valor de K(13); d) C4.5 com confiança igual a 0,1 e número mínimo de instâncias igual a 30; e e) Naive Bayes com o parâmetro useKernelEstimator igual a True.

Com base na Tabela 25, observa-se que, para a classificação considerando os sete gêneros, nenhum dos cenários apresentou taxa de acerto superior a 50,00\%; para esta estratégia, o melhor resultou foi obtido por meio da combinação entre a estratégia Boosting e o algoritmo Random Forest considerando 100 iterações $(48,00 \%)$. A matriz de confusão para este cenário é elencada na Tabela 26.

Tabela 26: Matriz de confusão Boosting (7 gêneros)

\begin{tabular}{cccccccc}
\hline & SAM & ROC & POP & MPB & JAZ & HM & MC \\
\hline SAM & $\mathbf{4 8}$ & 11 & 17 & 40 & 27 & 4 & 3 \\
ROC & 11 & $\mathbf{5 1}$ & 19 & 12 & 12 & 33 & 12 \\
POP & 20 & 15 & $\mathbf{7 3}$ & 17 & 13 & 8 & 4 \\
MPB & 55 & 12 & 9 & $\mathbf{4 7}$ & 23 & 2 & 2 \\
JAZ & 24 & 13 & 11 & 20 & $\mathbf{6 1}$ & 2 & 19 \\
HM & 5 & 25 & 6 & 8 & 8 & $\mathbf{9 2}$ & 6 \\
MC & 3 & 5 & 1 & 1 & 8 & 0 & $\mathbf{1 3 2}$ \\
\hline
\end{tabular}

Fonte: Elaborado pelos Autores (2019).

Conforme verifica-se na Tabela 26, os gêneros Música Clássica (88,00\%) e Heavy Metal (61,33\%) foram os únicos com taxa de acerto superior a $50,00 \%$; neste cenário, o gênero MPB teve apenas $31,33 \%$ dos seus registros classificados corretamente.

Com relação à classificação com cinco gêneros, observa-se na Tabela 25 que o melhor resultado em termos de acerto foi obtido ao utilizar-se o algoritmo Random Forest com o valor de 100 iterações $(62,40 \%)$. Este cenário é representado na Tabela 27.

Conforme exposto na Tabela 27, destaca-se os resultados obtidos para os gêneros Música Clássica $(87,33 \%)$, Jazz $(62,67 \%)$ e POP $(60,67 \%)$. Dos cinco gêneros,
Tabela 27: Matriz de confusão Boosting ( 5 gêneros)

\begin{tabular}{cccccc}
\hline & ROC & POP & JAZ & HM & MC \\
\hline ROC & 65 & 29 & 18 & 27 & 11 \\
POP & 22 & $\mathbf{9 1}$ & 25 & 9 & 3 \\
JAZ & 17 & 21 & 94 & 2 & 16 \\
HM & 33 & 11 & 11 & $\mathbf{8 7}$ & 8 \\
MC & 7 & 0 & 11 & 1 & 131 \\
\hline
\end{tabular}

Fonte: Elaborado pelos Autores (2019).

neste cenário, apenas o gênero Rock $(43,00 \%)$ ficou abaixo dos 50,00\% de acerto.

Quanto à classificação considerando apenas os gêneros Samba e MPB, o melhor resultado consistiu na combinação do algoritmo Boosting com o algoritmo C4.5, utilizando confiança de 0,1 e considerando o número mínimo de instâncias igual a 30. Esta combinação resultou em $65,00 \%$ de instâncias corretamente classificadas. Os resultados deste cenário são elencados na Tabela 28.

Tabela 28: Matriz de confusão Boosting (2 gêneros)

\begin{tabular}{ccc}
\hline & SAM & MPB \\
\hline SAM & 99 & 51 \\
MPB & 54 & 96
\end{tabular}

Fonte: Elaborado pelos Autores (2019).

Com base na Tabela 28, tem-se uma taxa de acerto de $66,00 \%$ para o gênero Samba e $64,00 \%$ de instâncias classificadas corretamente para o gênero MPB.

\subsection{Síntese dos resultados}

Comparando os resultados obtidos para a classificação dos sete gêneros musicais, percebe-se que, apenas ao utilizar-se a estratégia Bagging (50,85\%), combinada com o algoritmo Bayes Net foi possível obter-se uma taxa de acerto superior a 50,00\%. A síntese dos resultados para esta estratégia é representada na Tabela 29.

\begin{tabular}{|c|c|c|}
\hline Algoritmos & Parâmetros & Taxa de acerto \\
\hline $\mathrm{RF}$ & I $(400)$ & $49,05 \%$ \\
\hline $\mathrm{BN}$ & SE e TAN & $45,43 \%$ \\
\hline KNN & K (15) & $38,48 \%$ \\
\hline $\mathrm{C} 4.5$ & $\mathrm{C}(0,1)$ e mNO $(30)$ & $40,86 \%$ \\
\hline NB & useKernelE. (True) & $46,95 \%$ \\
\hline Bagging & Bayes Net & $50,85 \%$ \\
\hline Boosting & RF (100) & $48,00 \%$ \\
\hline
\end{tabular}

Fonte: Elaborado pelos Autores (2019).

Com relação aos resultados obtidos para a classificação dos cinco gêneros musicais, verifica-se um desempenho superior à estratégia considerando todos os gêneros. Neste cenário, todos os resultados apresentaram taxa de acerto superior a 50,00\%, com destaque, novamente, para a combinação da estratégia de Bagging com o algoritmo Random Forest - com 500 iterações -, cujo total de instâncias classificadas corretamente foi 
de $62,67 \%$. A síntese dos resultados para esta estratégia é representada na Tabela 30.

Tabela 30: Síntese dos resultados (5 gêneros)

\begin{tabular}{lcc}
\hline Algoritmos & Parâmetros & Taxa de acerto \\
\hline RF & I (500) & $62,53 \%$ \\
BN & SE e TAN & $59,47 \%$ \\
KNN & K (13) & $51,60 \%$ \\
C4.5 & C(0,1) e mNO (30) & $52,13 \%$ \\
NB & useKernelE. (True) & $59,67 \%$ \\
Bagging & RF (500) & $62,67 \%$ \\
Boosting & RF (100) & $62,40 \%$ \\
\hline
\end{tabular}

Fonte: Elaborado pelos Autores (2019).

Por fim, no tocante aos resultados obtidos para a classificação considerando apenas os gêneros Samba e MPB, nota-se um desempenho superior à estratégia considerando todos os gêneros. Neste cenário, todos os resultados apresentaram taxa de acerto superior a $50,00 \%$, com destaque, para o algoritmo $\mathrm{C} 4.5$ - com valor de 0,1 para o parâmetro de confiança e de 30 para o número mínimo de instâncias. $O$ total de instâncias classificadas corretamente correspondeu a $66,67 \%$. A síntese dos resultados para esta estratégia é representada na Tabela 31.

Tabela 31: Síntese dos resultados (2 gêneros)

\begin{tabular}{lcc}
\hline \multicolumn{1}{c}{ Algoritmos } & Parâmetros & Taxa de acerto \\
\hline RF & I (500) & $57,33 \%$ \\
BN & SE e TAN & $60,27 \%$ \\
KNN & K (13) & $57,33 \%$ \\
C4.5 & C(0,1) e mNO (30) & $66,67 \%$ \\
NB & useKernelE. (True) & $60,67 \%$ \\
Bagging (C4.5) & C(0,1) e mNO (30) & $62,00 \%$ \\
Boosting (C4.5) & C(0,1) e mNO (30) & $65,00 \%$ \\
\hline
\end{tabular}

Fonte: Elaborado pelos Autores (2019).

Observando-se os resultados dispostos nas Tabela 29 e ????, tem-se que, das três estratégias propostas, duas - sete e cinco gêneros - tiveram os melhores resultados mediante a aplicação da estratégia Bagging. Contudo, o melhor resultado foi obtido para a classificação binária entre os gêneros Samba e MPB, por meio do algoritmo C4.5, cujo resultado foi de $66,67 \%$ de acerto.

\subsection{Discussão a respeito dos erros de classifi- cação}

Diante do desempenho em alguns cenários envolvendo gêneros como o Samba, a MPB e o Rock, nota-se elementos em aberto no que condiz com a criação de limites entre determinados gêneros musicais (Goienetxea et al., 2018), uma vez que estes podem apresentar fronteiras amplas e nebulosas, representadas por características culturais (McKay and Fujinaga, 2006).

Com relação aos gêneros Samba e MPB, verificou-se uma "dificuldade" em diferenciá-los durante os experimentos. Esta "dificuldade" também se manifesta por parte dos usuários da plataforma Last.Fm, posto que, artistas como Chico Buarque, Novos Baianos, Marisa Monte, Maria Ritta e Cartola têm canções listadas em ambos os gêneros.

Em termos percentuais, por exemplo, para o gênero Samba, 32,67\% dos registros do gênero foram classificados equivocadamente como MPB no cenário em que se empregou o algoritmo Naive Bayes, considerando os sete gêneros musicais (Tabela 18). No cenário obtido utilizando o algoritmo Random Forest, 29,33\% das instâncias foram classificadas como MPB em um cenário com os sete gêneros (Tabela 2).

No que diz respeito ao gênero MPB, no cenário elaborado com o algoritmo KNN, 40,00\% dos registros foram classificados como Samba (Tabela 10), e 36,37\% considerando a combinação da estratégia Boosting com o algoritmo C.45 (Tabela 26).

Tal característica pode ter dificultado a descoberta de padrões capazes de diferenciar os gêneros em questão. Esta hipótese pode estar relacionada ao fato da MPB ter dialogado e absorvido elementos de outros gêneros musicais, como o Choro, gêneros regionais e o próprio Samba (Stroud, 2016).

Em determinados cenários, os gêneros Samba e MPB apresentaram registros associados ao gênero Jazz. Em questões percentuais, em dois cenários - Bayes Net com sete gêneros (Tabela 6) e $\mathrm{C} 4.5$ com sete gêneros (Tabela 14) - 20,00\% das instâncias do gênero Samba foram classificadas como Jazz. Já para as instâncias originalmente pertencentes ao gênero Jazz, ao se utilizar o algoritmo $\mathrm{KNN}, 34,67 \%$ dos registros foram classificados como Samba (Tabela 10).

Para o gênero MPB, destaca-se o desempenho para o algoritmo Naive Bayes. Com este algoritmo, 20,67\% das músicas originalmente classificadas como MPB foram classificadas como Jazz (Tabela 18). Por sua vez, com o mesmo algoritmo, 18,67\% das músicas de Jazz foram rotuladas como MPB (Tabela 18).

Tal comportamento tende a ser reflexo da relação de influência mútua entre estes gêneros, manifestada, por exemplo, pela incorporação de elementos da Música Popular Brasileira - principalmente relacionados à Bossa Nova - por parte dos músicos de Jazz, bem como o diálogo dos músicos brasileiros com o próprio Jazz (Garcia, 2018).

No que concerne ao gênero Rock, por este ser um gênero que abrange vários subgêneros (Silver et al., 2016, Shi et al., 2018), verificou-se registros associados ao gênero Heavy Metal ao longo dos cenários elaborados.

Ao se considerar uma classificação com sete gêneros, $26,67 \%$ dos registros do gênero Rock foram classificados como Heavy Metal (Tabela 18), enquanto 26,67\% das músicas do gênero Heavy Metal foram rotuladas como Rock (Tabela 10).

Ao se reduzir o número de gêneros para cinco, obteve-se $29,33 \%$ de registros de Rock classificados como Heavy Metal, por meio do algoritmo Naive Bayes (Tabela 19). Por sua vez, no tocante ao gênero Heavy Metal, o algoritmo KNN classificou 38,00\% deste gênero como Rock (Tabela 11).

Semelhante à relação "Samba-MPB", diversos artistas foram classificados pelos membros da plataforma Last.FM nos gêneros Rock e Heavy Metal, ao mesmo 
tempo. Dentre estes artistas, estão bandas como AC/DC, Black Sabbath, Deep Purple, Metallica e Scorpions.

\section{Considerações Finais}

A classificação de gêneros musicais apresentou-se como um "problema interessante" para trabalhos relacionados à aplicação de estratégias de Machine Learning, posto que, a existência de gêneros formados a partir da combinação de elementos de outros gêneros tende a dificultar a diferenciação entre eles.

Neste estudo, percebeu-se que, ao retirar-se os gêneros MPB e Samba, os resultados obtidos considerando uma classificação com apenas cinco gêneros e uma classificação binária (Samba e MPB) apresentaram-se superiores à estratégia com os sete gêneros, reforçando e justificando a opção pelas estratégias de classificação empregadas.

No que tange aos gêneros musicais, destaca-se o desempenho de classificação para a Música Clássica; em todos os experimentos demonstrou os melhores índices de acerto, com valores acima dos 70,00\%. Em contrapartida, os demais gêneros musicais não apresentaram um padrão nos resultados, variando conforme o método aplicado.

Com relação aos algoritmos empregados, destaca-se a combinação entre os algoritmos Bagging e Bayes Net, que resultou em $50,85 \%$ de acerto para a classificação com sete gêneros, bem como a combinação dos algoritmos Bagging e Random Forest, que alcançou 62,67\% de acerto para a classificação com cinco gêneros musicas. Por sua vez, o algoritmo $\mathrm{C} 4.5$ obteve o melhor resultado de todos, com $66,67 \%$ de instâncias classificadas corretamente para o cenário entre Samba e MPB.

Como trabalhos futuros, incentiva-se a utilização de gêneros musicais adicionais. A música eletrônica, por exemplo, por apresentar características diferentes dos gêneros aqui empregados pode ser um gênero interessante para efeitos de comparação. Estimula-se considerar descritores como Linear Predictive Coding (LPC) e a utilização dos 13 Coeficientes Cepstrais da frequência Mel como acréscimo aos descritores utilizados neste estudo.

Sugere-se o acréscimo de elementos relacionados às letras das canções, às progressões de acordes e às emoções aos descritores relacionados ao áudio. Acredita-se que estes elementos tendem a contribuir na distinção de gêneros que apresentem possíveis fronteiras não tão bem definidas.

Finalmente, sugere-se a realização de novos experimentos com algoritmos baseados em redes neurais e algoritmos genéticos, e recomenda-se a utilização de arquivos com duração de 45 segundos e 20 segundos cada a fim de elaborar comparações entre os resultados obtidos para diferentes durações dos registros.

\section{Referências}

Baniya, B. K. and Lee, J. (2016). Importance of audio feature reduction in automatic music genre classifi- cation, Multimedia Tools and Applications 75(6): 30133026. https://doi.org/10.1007/s11042-014-2418-z.

Breiman, L. (1996). Bagging predictors, Machine Learning 24(2): 123-140. https://doi.org/10.1007/ BF00058655.

Breiman, L. (2001). Random forests, Machine Learning 45(1): 5-32. https://doi.org/10.1023/A: 1010933404324.

Cheng, Z., Shen, J., Nie, L., Chua, T.-S. and Kankanhalli, M. (2017). Exploring user-specific information in music retrieval, Proceedings of the 4oth International ACM SIGIR Conference on Research and Development in Information Retrieval, ACM, Shinjuku, Tokyo, Japan. https://doi.org/10.1145/3077136.3080772.

Correa, D. C. and Rodrigues, F. A. (2016). A survey on symbolic data-based music genre classification, Expert Systems with Applications 60: 190-210. https: //doi.org/10.1016/j.eswa.2016.04.008.

Costa, Y. M. G., Oliveira, L. S. and Silla Jr., C. N. (2017). An evaluation of convolutional neural networks for music classification using spectrograms, Applied Soft Computing 52: 28-38. https://doi.org/10.1016/j. asoc.2016.12.024.

Cover, T. M. and Hart, P. E. (1967). Nearest neighbor pattern classification, IEEE Transactions on Information Theory 13(1): 21-27. https : //doi .org/10.1109/TIT. 1967.1053964.

Deshpande, H., Singh, R. and Nam, U. (2001). Classification of music signals in the visual domain, Proceedings of the COST-G6 Conference on $\mathrm{Di}$ gital Audio Effects, Univ., Limerick,IE. Disponível em http://citeseerx.ist.psu.edu/viewdoc/download? doi=10.1.1.13.4634\&rep=rep1\&type=pdf.

Fayyad, U., Piatetsky-Shapiro, G. and Smyth, P. (1996). The kdd process for extracting useful knowledge from volumes of data, Commun. ACM 39(11): 27-34. http: //doi.acm.org/10.1145/240455.240464.

Freund, Y. and Schapire, R. E. (1997). A decisiontheoretic generalization of on-line learning and an application to boosting, Journal of Computer and System Sciences 55(1): 119-139. https://doi.org/10.1006/ jcss.1997.1504.

Friedman, N., Geiger, D. and Goldszmidt, M. (1997). Bayesian network classifiers, Machine Learning 29(2): 131-163. https://doi.org/10.1023/A: 1007465528199.

Garcia, M. (2018). Show opinião: quando a mpb entra em cena (1964-1965), Históra (São Paulo) 37: 1-33. http://dx.doi.org/10.1590/1980-4369e2018036.

Goienetxea, I., Martínez-Otzeta, J. M., Sierra, B. and Mendialdua, I. (2018). Towards the use of similarity distances to music genre classification: A comparative study, PLOS ONE 13(2): 1-18. https: //doi .org/10. 1371 /journal.pone. 0191417. 
Grimaldi, M., Cunningham, P. and Kokaram, A. (2003). A wavelet packet representation of audio signals for music genre classification using different ensemble and feature selection techniques, Proceedings of the 5th ACM SIGMM International Workshop on Multimedia Information Retrieval, ACM, New York,USA. https: //doi.org/10.1145/973264.973281.

Huang, Y.-F., Lin, S.-M., Wu, H.-Y. and Li, Y.-S. (2014). Music genre classification based on local feature selection using a self-adaptive harmony search algorithm, Data \& Knowledge Engineering 92: 60-76. https://doi.org/10.1016/j.datak.2014.07.005.

Kotsifakos, A., Kotsifakos, E. E., Papapetrou, P. and Athitsos, V. (2013). Genre classification of symbolic music with smbgt, Proceedings of the 6th International Conference on PErvasive Technologies Related to Assistive Environments, ACM, New York,USA. https://doi.org/ $10.1145 / 2504335.2504382$.

Li, T., Ogihara, M. and Li, Q. (2003). A comparative study on content-based music genre classification, Proceedings of the 26th Annual International ACM SIGIR Conference on Research and Development in Informaion Retrieval, ACM, Toronto,CAN. https://doi.org/ 10.1145/860435.860487.

Mcadams, S. (1999). Perspectives on the contribution of timbre to musical structure, Comput. $\mathrm{Mu}$ sic J. 23(3): 85-102. http://dx.doi.org/10.1162/ 014892699559797.

McKay, C. (2010). Automatic Music Classification with jMIR, Ph.d. - music technology, McGill University, Montreal, Canada.

McKay, C. and Fujinaga, I. (2006). Musical genre classification: Is it worth pursuing and how can it be improved?, Proceedings of the 7th International Society of Music Information Retrieval Conference (ISMIR 2006), ISMIR, Vitoria, Canadá. Disponível em https: //ismir2006.ismir.net/PAPERS/ISMIR06109_Paper.pdf.

Murphy, Y. V. S. and Koolagudi, S. G. (2018). Contentbased music information retrieval (cb-mir) and its applications toward the music industry: A review, ACM Comput. Surv. 51(3): 45:1-45:46. http://doi.acm. org/10.1145/3177849.

Nanni, L., Costa, Y. M. G., Lumuni, A., Kim, M. Y. and Baek, S. R. (2016). Combining visual and acoustic features for music genre classification, Expert Systems with Applications 45: 108-117. https://doi.org/ 10.1016/j. eswa. 2015.09.018.

Oramas, S., Nieto, O., Barbieri, F. and Serra, X. (2017). Multi-label music genre classification from audio, text, and images using deep features, Proceedings of the 18th International Society of Music Information Retrieval Conference (ISMIR 2017), ISMIR, Suzhou, China. Disponível em https://arxiv.org/abs/1707.04916.

Quinlan, J. R. (1993). C4.5: programs for machine learning, Morgan Kaufmann.
Romano, L. E. and Adami, A. G. (2015). Reconhecimento automático de gêneros musicais utilizando classificadores baseados em múltiplas características, Revista Brasileira de Computação Aplicada 7(1): 85-99. https://doi.org/10.5335/rbca.2015.4281.

Rosner, A. and Kostek, B. (2017). Automatic music genre classification based on musical instrument track separation, Journal of Intelligent Information Systems 50(2): 363-384. https://doi.org/10. 1007/s10844-017-0464-5.

Shi, Y., Lim, Y. and Suh, C. S. (2018). Innovation or deviation? the relationship between boundary crossing and audience evaluation in the music field, PLOS ONE 13(10): 1-17. https://doi.org/10.1371/journal. pone. 0203065.

Silla Jr., C. N., Kaestner, C. A. A. and Koerich, A. L. (2005). Classificação automática de gêneros musicais utilizando métodos de bagging e boosting, 1oth Brazilian Symposium on Computer Music, SBC, Belo Horizonte, Minas Gerais, BR. Disponível em http://compmus.ime.usp.br/sbcm/2005/papers/ tech-12438.html.

Silver, D., Lee, M. and C., C. C. (2016). Genre complexes in popular music, PLOS ONE 11(5): 1-23. https://doi. org/10.1371/journal pone.0155471.

Stone, M. (1974). Cross-validatory choice and assessment of statistical predictions, Journal of the Royal Statistical Society. Series B (Methodological) 36(2): 111-147. Disponível em http://www. jstor.org/stable/2984809.

Stroud, S. (2016). The Defence of Tradition in Brazilian Popular Music Politics, Culture and the Creation of Música Popular Brasileira, Routledge.

Tang, C. P., Chui, K. L., Zeng, Z. and Wong, K. H. (2018). Music genre classification using a hierarchical long short term memory (lstm) model, Proceedings Volume 10828, Third International Workshop on Pattern Recognition; 108281B (2018), Jinan, CHN. https://doi. org/10.1117/12.2501763.

Tzanetakis, G. and Cook, P. (2002). Musical genre classification of audio signals, IEEE Transactions on Speech and Audio Processing 10(5): 293-302. https: //doi.org/10.1109/TSA.2002.800560.

Wu, M.-J. and Jang, J.-S. R. (2015). Combining visual and acoustic features for music genre classification, ACM Trans. Multimedia Comput. Commun. Appl. 12(1): 10:1-10:17. http://doi.acm.org/10.1145/ 2801127. 